\section{Cytokinin-containing Seaweed Extract Does Not Reduce Damage by an Insect Herbivore}

\author{
Stuart R. Reitz and John T. Trumble \\ Department of Entomology, University of California, Riverside, CA 92521
}

Additional index words. tomato, Lycopersicon esculentum, lima bean, Phaseolus lunatus, beet armyworm, Spodoptera exigua, Ascophyllum nodosum, growth inhibition

\begin{abstract}
We examined two aspects of treating plants with a cytokinin-containing seaweed extract (SWE). In the first series of experiments, we tested the hypothesis that immature lima bean (Phaseolus lunatus L.) and tomato (Lycopersicon esculentum Mill.) plants provided with exogenous cytokinins could recover from defoliation by a generalist insect herbivore, Spodoptera exigua (Hübner), more rapidly than plants without cytokinin supplements. However, the SWE inhibited growth of lima beans at all levels of herbivore damage. The SWE neither inhibited nor stimulated growth of tomatoes following defoliation. Because SWE effects largely were neutral for tomato growth, we conducted a second series of experiments to test the hypothesis that SWE treatments alter the attractiveness of tomato foliage to $S$. exigua larvae. In these experiments, we determined consumption of, and preference for, SWE-treated tomato foliage by $S$. exigua larvae. Repeated root applications of SWE led to increased consumption and preference by $S$. exigua. Repeated foliar applications did not alter consumption or preference compared with controls. Spodoptera exigua larvae gained significantly more mass when feeding on SWE-treated foliage compared with controls. While these data indicate that plant responses to exogenous cytokinin-containing materials depend on taxa and application method, the practical uses of SWE appear limited given the negative effects on plant growth and increased attractiveness of treated foliage to herbivores.
\end{abstract}

Traditionally, integrated pest management programs have emphasized maintaining pest populations below levels that would result in economic damage to crops. A complementary approach to this type of strategy is to increase the ability of crops to recover from herbivore damage, thereby increasing economic injury levels. One potential method for stimulating plant recovery from herbivore damage is exogenous applications of plant hormones that stimulate growth. Among the most important phytohormones affecting growth are cytokinins. Cytokinins promote cell division and expansion (Elliott, 1982; Taiz and Zeiger, 1991; Weaver, 1972) and inhibit senescence (Nooden and Leopold, 1978), and they are important in wound responses as they occur at high levels in leaves of plants recovering from herbivory (Trumble et al., 1993) or artificial damage (Palmer et al., 1981; Wang et al., 1977). Large-scale applications of these compounds, however, are prohibitively expensive. Liquid extracts of seaweeds (SWE), which contain relatively high endogenous levels of cytokinins, reportedly result in increased growth of a variety of crop plants (Beckett

\footnotetext{
Received for publication 30 May 1995. Accepted for publication 30 Sept. 1995. We appreciate the technical assistance of Mariano Berdegue, William Carson, Cesar Rodriguez, and Kristina White. The cost of publishing this paper was defrayed in part by the payment of page charges. Under postal regulations, this paper therefore must be hereby marked advertisement solely to indicate this fact.
}

and van Staden, 1991; Mooney and van Staden, 1985; Senn et al., 1961), although not in all cases (Blunden and Wildgoose, 1977; Crouch and van Staden, 1994; Hedin and McCarty, 1994; Humphries, 1958). Therefore, under appropriate circumstances, exogenous applications of cytokinins or cytokinin-containing compounds may increase recovery of plants from herbivore damage. In addition, these SWE also reduced pest damage in certain systems (Booth, 1966), but not all (Pakarinen et al., 1990). Given the reported benefits of cytokinin-containing SWE to crop plants, we tested the hypotheses that SWE increase plant recovery following defoliation and that SWE alter the palatability of foliage to herbivores.

The two plant systems we used to test the effect of SWE on plant compensation were immature lima beans and tomatoes. We used tomatoes to examine the response of herbivores to SWE-treated plants. Larvae of the beet armyworm, a generalist insect herbivore, were used in all experiments.

\section{PLANT GROWTH FOLLOWING DEFOLIATION}

\section{Materials and Methods}

Cytokinin extract. We used a commercially available extract prepared from the marine brown alga Ascophyllum nodosum L. (Cytokin, Plant Biotech, Corrales, N.M.) that has a cytokinin activity equivalent to $0.01 \% \mathrm{ki}$ netin. The treatment for all tests was a manufacturer-suggested rate of $4 \mathrm{ml} \cdot \mathrm{liter}^{-1}$ in an aqueous solution of $0.1 \%$ Tween 80 . Control plants were treated with a solution lacking the A. nodosum extract.

Plant material. Lima beans ('Henderson Bush') were germinated in vermiculite-filled tubs and transplanted individually into pots $(10 \times 10 \mathrm{~cm}, 0.4$ liter $)$ containing a soil mixture (Matkin and Chandler, 1957), when primary leaves of plants had unfolded $(\approx 7$ days after germination). Plants were maintained in a greenhouse under ambient conditions at the Univ. of California, Riverside. The experiment was run twice, with plants in the first receiving no supplemental fertilization while those in the second were fertilized weekly with a $15 \mathrm{~N}-12.9 \mathrm{P}-12.5 \mathrm{~K}$ solution $\left(3 \mathrm{mg} \cdot \mathrm{ml}^{-1}\right)$ (Stern's Miracle Grow, Port Washington, N.Y.). Experiments began when the first trifoliate leaves were expanded fully.

Tomatoes (UCD-82) were germinated in flats containing a soil mixture (Matkin and Chandler, 1957) and were transplanted to individual pots $(12.5 \mathrm{~cm}$ in diameter, 1 liter $)$ containing a mixture of 1 soil : 1 peat : 1 vermiculite when $\approx 10 \mathrm{~cm}$ tall. Tomatoes were grown under greenhouse conditions with supplemental lighting (14-h light/10-h dark photoperiod). Tomatoes were fertilized weekly with a $15 \mathrm{~N}-12.9 \mathrm{P}-12.5 \mathrm{~K}$ solution. Experiments began when plants averaged $16.5 \mathrm{~cm}$ $(\mathrm{SD}=2.0)$ tall and $5.5(\mathrm{SD}=1.0)$ leaf-bearing stems.

Insect herbivore damage and SWE treatment. Newly molted fourth instars of S. exigua obtained from a laboratory colony were used for all tests. The laboratory colony was reared on artificial medium (Shorey and Hale, 1965) and had new field-collected material added within 6 months of when testing began. Plants $(n=12$ per treatment combination) were enclosed in nylon mesh bags and received one of three herbivore densities $(0,1$, or 3 larvae per plant). Larvae fed on lima beans for $48 \mathrm{~h}$ or on tomatoes for $24 \mathrm{~h}$ before removal. Herbivore damage to plants was estimated by counting the number of damaged and undamaged leaves per plant immediately after larvae were removed from plants. The day following herbivore removal, plants were sprayed to runoff with a manual atomizer with either the SWE or control solution. These applications were repeated three more times at weekly intervals for a total of four treatments. Plants were harvested 1 week after the final application.

Data collection and analysis. At harvest, we measured several relevant plant growth characteristics. Maximum stem height was recorded at harvest, and change in stem length was recorded as the difference between final stem height and stem height after herbivore removal. Aboveground plant components were separated into stems and leaves. Wet mass of these components was recorded, and leaf area was determined with a leaf area meter (model 3000; LI-COR, Lincoln, Neb.). Dry mass of leaves and stems were recorded after drying for 1 week at $65 \mathrm{C}$

Data were tested by analysis of covariance, with the SuperAnova statistical package (Abacus Concepts, Berkeley, Calif.). Initial stem 
height was included as a covariate in models to account for differences among plants. Pairwise comparisons between SWE treatments and their respective controls are based on leastsquares means $t$ tests. Means and SES are reported.

\section{Results}

For both lima beans and tomatoes, there was a clear distinction among S. exigua densities in the amount of plant damage. Plants with three larvae per plant had the more severe damage, with $>50 \%$ of leaves substantially damaged, whereas those with one larva per plant had a more moderate level of leaves damaged $(\approx 25 \%)$. There were no significant interactions between $S$. exigua density and SWE treatment for either lima beans or tomatoes $(P>0.05)$.

Lima beans. In general, foliar applications of the SWE resulted in smaller plants that did not produce as much new growth as control plants. Although plants receiving supplemental fertilizer were larger than unfertilized plants, there was no interaction between fertilizer and other treatments $(P>0.05)$. In terms of stem height, SWE-treated plants grew less than controls following herbivore damage $(P<0.001$; Fig. 1a). A growth retardant effect was evident as undamaged and moderately damaged (one larva per plant) lima beans actually lost height over the course of the test. Also, these SWE-treated plants had significantly less leaf area than the control plants $(P<0.0001$, Fig. 1b). Because SWE-treated plants added significantly less new leaf area following herbivore damage $(P<0.0001$, Fig. $1 b$ ), differences in total leaf area are attributable to the effect of the SWE and not any possible differences in the amount of herbivore damage. The effect of the SWE on wet and dry masses $(P<0.01$, Fig. 1c) of lima beans followed the same pattern as for stem length and leaf area, with treated plants consistently being significantly smaller than controls.

Other physiological effects on lima beans indicate that the SWE increases the allocation of resources to existing tissues at the expense of the plants producing new growth. Leaf wet mass per unit area and leaf dry mass per unit area were greater for SWE-treated lima beans than for controls $(P<0.0001$, Fig. 1d).

Tomatoes. The effects of the SWE on tomatoes were not as pronounced as with lima beans. The SWE did not inhibit growth to a great extent, but neither did it promote new growth. Overall, SWE-treated tomatoes did not grow as tall as controls following herbivore damage $(P<0.05$, Fig. $2 \mathrm{a})$; however, controls did not produce more leaf area than SWE-treated tomatoes $(P>0.05$, Fig. 2b). There was no significant difference in wet or dry mass of SWE-treated and nontreated tomatoes $(P>0.05$, Fig. 2c). This lack of difference between SWE treatments can in part be attributed to the greater leaf wet mass per unit area and leaf dry mass per unit area of SWEtreated tomatoes compared to controls $(P<0.05$, Fig. $2 \mathrm{~d})$.
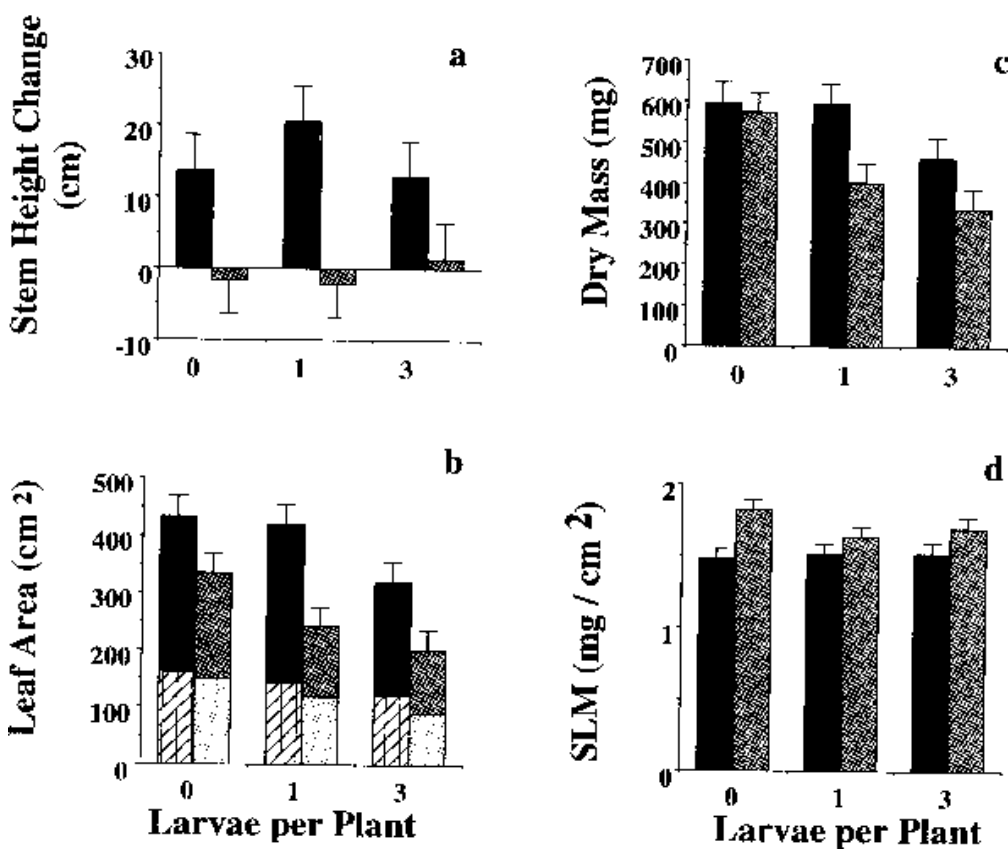

Fig. 1. Effects of a cytokinin-containing seaweed extract (SWE) on growth characteristics of lima beans following partial defoliation by Spodoptera exigua. Columns represent means and associated SEs for (ם) control and ( $\square$ ) SWE treatments ( $\mathrm{n}=12$ per treatment). (a) Stem height, (b) total leaf area (represented by the entire bar) and area added following herbivory (darker shaded, upper portion of bars), and (c) dry mass were significantly lower for SWE-treated plants compared with controls $(P<0.05$, analysis of variance). (d) Specific leaf mass (SLM) was significantly higher for SWEtreated plants compared with controls $(P<0.05)$
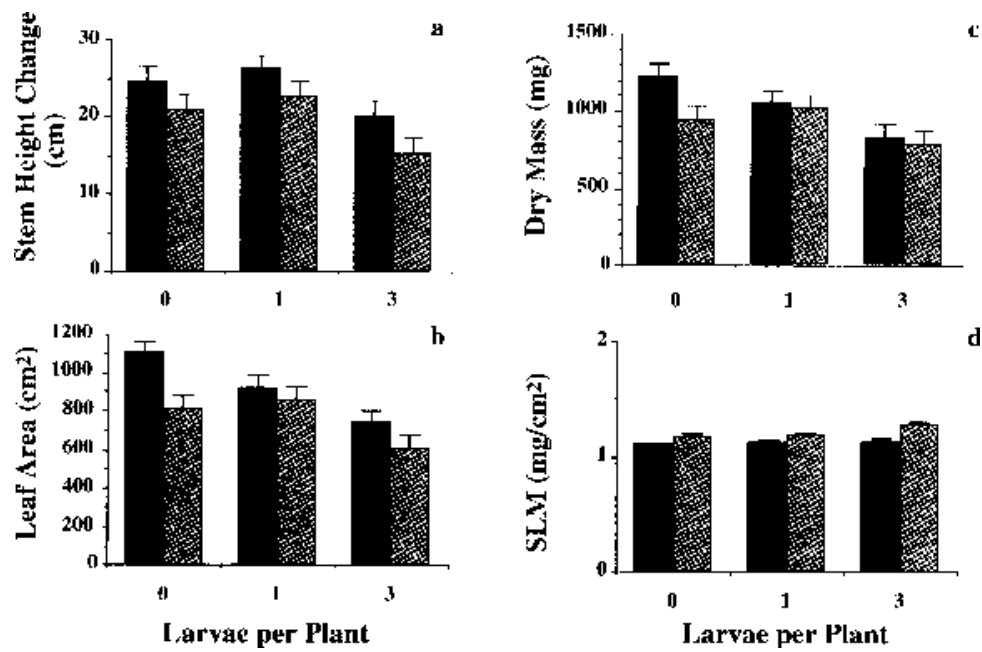

Fig. 2. Effects of a cytokinin-containing seaweed extract (SWE) on growth characteristics of tomatoes following partial defoliation by Spodoptera exigua. Columns represent means and associated sEs for (ם) control and ( $\square$ ) SWE treatments ( $=12$ per treatment). (a) Stem height was significantly lower for SWEtreated plants compared with controls $(P<0.05$, analysis of variance). (b) Total leaf area and (c) dry mass were not significantly different between SWE-treated and control plants $(P>0.05)$. (d) Specific leaf mass (SLM) was significantly greater for SWE-treated plants compared with controls $(P<0.05)$.

\section{SWE EFFECTS ON HERBIVORE CONSUMPTION AND PREFERENCE OF TOMATO FOLIAGE}

\section{Materials and Methods}

Four SWE treatments were applied to tomatoes in these experiments. Tomatoes received either repeated foliar applications of SWE, root drench applications of SWE, control treatments, or one foliar application immediately before presentation to larvae. These treatments allowed us to determine if differences in consumption or preference of foliage result from type of application (foliar vs. root), SWE-induced physiological changes in tomatoes (foliar and root vs. control), or a direct effect of the SWE itself.

SWE solutions were prepared as previously described. Two varieties of tomatoes (VFN-7718 and UCD-82) were used. Tomato plants were grown as previously described. In the control treatment, plants received four weekly foliar applications of a $0.1 \%$ Tween 80 aqueous solution (see previous experiment). For the repeated foliar application, plants received four weekly applications of the SWE solution. For the root drench, plants received 
four weekly applications of the SWE solution (50 $\mathrm{ml} /$ application). These three treatments began when plants were $\approx 20 \mathrm{~cm}$ tall. Foliage had not been sprayed for 1 week before testing with larvae was initiated. Plants in the direct treatment were treated $2 \mathrm{~h}$ before exposure to larvae, when foliage was sprayed to runoff with the SWE solution. All foliage was taken from the middle third of the plant canopy, thus standardizing the SWE effect and minimizing variation in leaf age and quality.

Consumption tests. In consumption tests, larvae (one per dish) were given an excess amount of foliage on which to feed. Two leaflets (total area $\approx 50 \mathrm{~cm}^{2}$ ) were placed on opposite sides of a petri dish $(15 \mathrm{~cm}$ in diameter) that was partially filled with agar to maintain moisture. Area of all foliage was measured before and after presentation to larvae. Similarly, larvae were weighed before and after testing. Consumption tests were conducted for $48 \mathrm{~h}$, with the original foliage being removed and replaced with fresh foliage after $24 \mathrm{~h}$. Data for leaf area consumed and larval mass change were analyzed as a factorial experiment with the two cultivars and four SWE treatments $(n=14$ for each treatment $\times$ cultivar combination). Initial larval mass was included as a covariate. Means comparisons are based on least-squares means $t$ tests.

Preference tests. Preference tests were designed as paired comparisons among the four SWE treatments (total of six comparisons per cultivar). Two equally sized leaflets $(<5 \%$ difference in area between leaflets), one each of two SWE treatments, were placed on opposite sides of an agar-lined petri dish. Then, a newly molted larva was placed in the middle of each dish. Larvae were allowed to feed for 24 $\mathrm{h}$, at which time they were removed and area consumed for each leaflet was determined as previously described. Differences in area consumed for each treatment were analyzed using paired $t$ tests ( $\mathrm{n}=15$ per comparison for UCD82 , and $n=24$ per comparison for VFN-7718).

\section{Results}

Results of these consumption and preference studies indicate that SWE effects depend on cultivar and application method. Spodoptera exigua larvae consumed more UCD-82 foliage than VFN-7718 foliage $(P<0.05)$ and differences between the two cultivars were consistent across the four SWE treatments. Therefore, comparisons among SWE treatments are limited to the overall effect. Spodoptera exigua larvae consumed more of the root-treated foliage than either the longterm foliar-treated foliage or control foliage (Fig. 3). The similarity in consumption between foliar-treated and control foliage is consistent with the minimal effect of SWE foliar applications on tomato growth following herbivory. While long-term foliar applications did not affect consumption, S. exigua larvae consumed significantly more of the foliage that had been sprayed immediately before testing compared to foliar applications and control applications $(P<0.01)$.

Spodoptera exigua larvae also gained sig- nificantly more mass when feeding on SWEtreated foliage $(P=0.01$, Fig. 4$)$. Despite the lack of a difference in consumption, S. exigua larvae gained more mass on the foliar-treated than on the control foliage. Therefore, the greater mass gain for $S$. exigua larvae feeding on SWE-treated foliage can be attributed to the increased specific leaf mass (dry mass per unit area) of treated foliage (foliar applications compared to controls), and the indirect and direct effect of the SWE on increasing the attractiveness of the foliage (root and direct applications).

Based on the consumption tests, we ex- pected $S$. exigua larvae to prefer root-drench and directly treated foliage more than foliage receiving repeated foliar applications or control applications. Root applications did greatly increase the attractiveness of VFN-7718 foliage to $S$. exigua larvae compared to control foliage $(P<0.05)$. Also, directly treated VFN7718 foliage was preferred compared with control foliage. For the UCD-82 plants, the only preference shown was for root-treated foliage compared to directly treated foliage. Given that larvae consumed the most foliage in these two treatments, the significantly greater preference for root-treated UCD-82 foliage

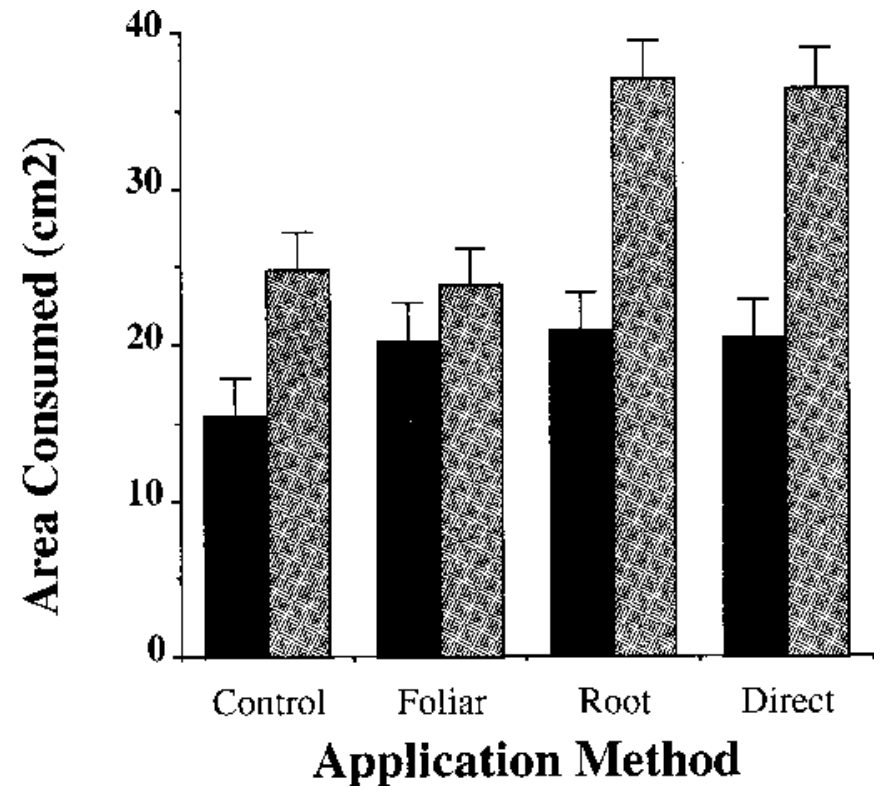

Fig. 3. Effect of cytokinin-containing seaweed extract (SWE) application method on consumption of tomato foliage (匹, VFN-7718; $\square$, UCD-82) by Spodoptera exigua. Columns represent means and associated SES for each application method ( $\mathrm{n}=14$ per method). The main effect for SWE treatment was significant $(P<0.05$, analysis of variance). See text for treatment comparisons.

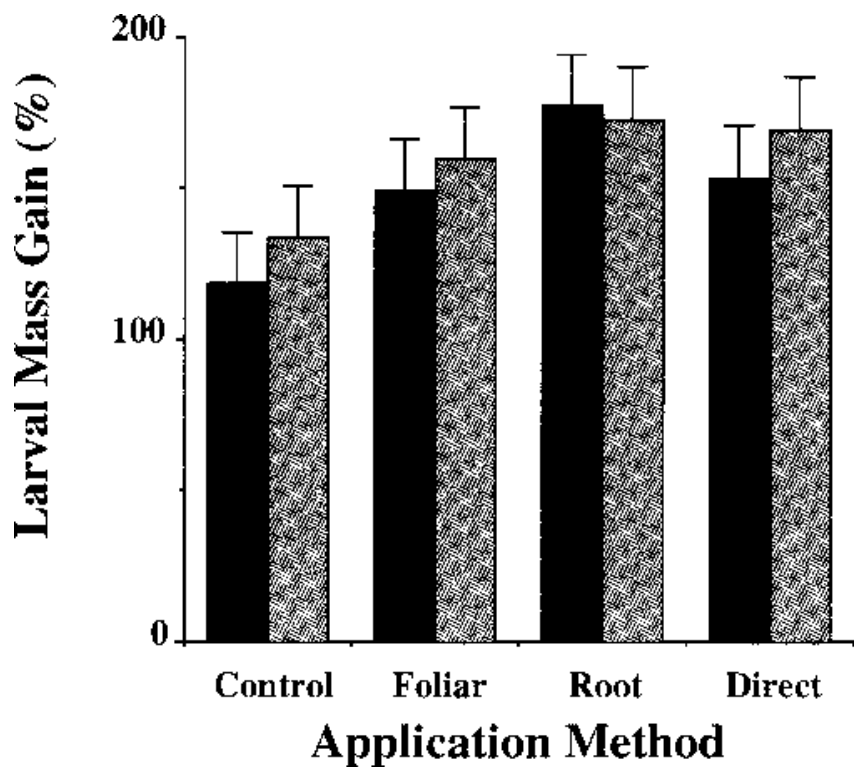

Fig. 4. Mass gain (percent increase of initial mass) of Spodoptera exigua larvae when feeding on cytokinincontaining, seaweed-extract (SWE)-treated tomato foliage (ם, VFN-7718; $\square$, UCD-82) for $48 \mathrm{~h}$. Columns represent means and associated sEs for each application method $(\mathrm{n}=14$ per treatment). The main effect for SWE treatment was significant $(P<0.05$, analysis of variance). See text for treatment comparisons. 
compared with directly treated foliage should be interpreted as an increased preference for root-treated foliage and not as a deterrent effect of the direct treatment.

\section{Discussion}

We were interested in examining two interactions of treating plants with a cytokinincontaining SWE and insect herbivory. The first effects we examined were those of postdamage applications of SWE on the recovery of plants. The second were the effects of pretreatment of plants with SWE on an insect herbivore. Neither effect warrants the inclusion of this particular SWE in the production methods of either plant system we examined.

Our results from the experiments examining recovery of lima beans and tomatoes from herbivore damage show that the SWE we used is biologically active for lima beans and tomatoes but does not enhance plant growth. The greater SLM of treated plants suggest that SWE caused plants to allocate more resources to existing tissue at the expense of producing new growth. Reallocation of resources in this way at the vegetative stage could delay flowering or fruit set. Except for this possibility, foliar applications of SWE largely were neutral in respect to tomato growth, in contrast to the strong inhibitory effects of SWE treatments on lima beans. Although effects of SWE can be dosage dependent (Crouch and van Staden, 1992; Featonby-Smith and van Staden, 1983), higher rates of SWE are even more inhibitory to lima beans (Reitz and Trumble, 1996). The differential effects of SWE that we observed for lima beans and tomatoes, as well as those of other studies (Crouch and van Staden, 1994), indicate that SWE effects are taxonomically dependent.

Most studies of the effects of SWE have focused on agronomic traits, such as growth and yield under controlled conditions. While SWE may produce desirable results in certain traits, other simultaneous effects may be counterproductive. We found that using SWE may not be suitable for lima beans because of its inhibitory effect on growth, but growth is only one aspect of production that needs to be considered. Similar cytokinin-containing materials have increased levels of the toxic allelochemical gossypol in some varieties of cotton (Gossypium hirsutum L.) (Hedin and McCarty, 1994). However, most of these increases in gossypol content were associated with high levels of exogenous cytokinin treatments that also tended to be associated with decreased cotton yields. Other SWE are toxic to two-spotted red spider mite, Tetranychus urticae Koch (Amer et al., 1991; Hankins and Hockey, 1990), but the concentrations of SWE Amer et al. (1991) applied to lima bean leaf disks were several times higher than concentrations that inhibit growth of lima beans. In a similar vein, infestations of root-knot nematodes, Meloidogyne incognita (Kofoid and White), on tomatoes can be reduced by root applications of SWE (Featonby-Smith and van Staden, 1983), but the SWE we tested could directly and indirectly increase foliar damage to tomatoes. Root applications of SWE to tomatoes resulted in greater consumption of foliage by $S$. exigua larvae. In addition, because $S$. exigua larvae gained more mass when feeding on treated foliage, future pest populations could be higher as fecundity of Spodoptera is correlated with size (Rothschild, 1969).

Discerning the effect of exogenous applications of phytohormones is complex. The effects of these phytohormones are mediated by their concentration and the physiological condition of the plant. Under certain circumstances, supplemental cytokinins may produce benefits, although we found no benefits under the conditions that we examined. In addition, benefits must be weighed against possible deleterious effects. Therefore, caution should be exercised in using SWE, with appropriate studies conducted on the possible consequences, before large-scale applications are attempted.

\section{Literature Cited}

Amer, S.A.A., N.Z. Dimertry, and A.S. Reda. 1991. Toxicity of green marine algae to the two-spotted spider mite Tetranychus urticae Koch. Insect Sci. Appl. 12:481-485.

Beckett, R.P. and J. van Staden. 1990. The effect of seaweed concentrate on the yield of nutrient stressed wheat. Bot. Marina 33:147-152.

Blunden, G. and P.B. Wildgoose. 1977. The effects of aqueous seaweed extract and kinetin on potato yields. J. Sci. Food. Agr. 28:121-125.

Booth, E. 1966. Some properties of seaweed manures. Proc. Intl. Seaweed Symp. 5:349-357.

Crouch, I.J. and J. van Staden. 1992. Effect of seaweed concentrate on the establishment and yield of greenhouse tomato plants. J. Appl. Phycol. 4:291-296.

Crouch, I.J. and J. van Staden. 1994. Commercial seaweed products as biostimulants in horticulture. J. Home Consumer Hort. 1:19-76.

Elliott, M.C. 1982. The regulation of plant growth, p. 57-98. In: T.H. Thomas (ed.). Plant growth regulator potential and practice. BCPC Publications, London.

Featonby-Smith, B.C. and J. van Staden. 1983. The effect of seaweed concentrate on the growth of tomato plants in nematode-infested soil. Scientia Hort. 20:137-146.
Hankins, S.D. and H.P. Hockey. 1990. The effect of a liquid seaweed extract from Ascophyllum nodosum (Fucales, Phaeophyta) on the 2-spotted red spider mite Tetranychus urticae. Hydrobiologia 204:555-559.

Hedin, P.A. and J.C. McCarty. 1994. Effects of several commercial plant growth regulator formulations on yield and allelochemicals of cotton (Gossypium hirsutum L.). J. Agr. Food Chem. 42:1355-1357.

Humphries, E.C. 1958. The effects of gibberellic acid and kinetin on the growth of Majestic potato. Ann. Appl. Biol. 46:346-351.

Matkin, O.A. and P.A. Chandler. 1957. The U.C.type soil mixes, p. 68-85. In: The U.C. system for producing healthy container-grown plants. California Agr. Expt. Sta Manual 23, Berkeley.

Mooney, P.A. and J. van Staden. 1985. Effect of seaweed concentrate on the growth of wheat under conditions of water stress. South Africa J. Sci. 81:632-633.

Nooden, L.D. and A.C. Leopold. 1978. Phytohormones and the endogenous regulation of senescence and abscission, p. 329-369. In: D.S. Letham, P.B. Goodwin, and T.J.V. Higgins. Phytohormones and related compounds: A comprehensive treatise. Elsevier, Amsterdam.

Pakarinen, E., P. Niemela, and J. Tuomi. 1990. Effect of fertilization, seaweed extracts and leafdamage on palatability of lettuce to Deroceras slugs. Acta Oecologica-Intl. J. Ecol. 11:113119.

Palmer, M.V., R. Horgan, and P.F. Wareing. 1981. Cytokinin metabolism in Phaseolus vulgaris $\mathrm{L}$. I. Variations in cytokinin levels in leaves of decapitated plants in relation to lateral bud outgrowth. J. Expt. Bot. 32:1231-1241.

Reitz, S.R. and J.T. Trumble. 1996. Effects of a cytokinin-containing seaweed extract on Phaseolus lunatus L.: Influence of nutrient availability and apex removal. Bot. Marina (In press.)

Rothschild, G.L.H. 1969. Observations on the armyworm Spodoptera mauritia acronyctoides Gn. (Lep., Noctuidae) in Sarawak (Malaysian Borneo). Bul. Entomol. Res. 59:143-160.

Senn, T.L., J.A. Martin, J.H. Crawford, and C.W. Derting. 1961. The effect of Norwegian seaweed (Ascophyllum nodosum) on the development and composition of certain horticultural and special crops. South Carolina Agr. Expt. Sta., Res. Serial 23.

Shorey, H.H. and R.L. Hale. 1965. Mass-rearing of the larvae of nine noctuid species on a simple artificial medium. J. Econ. Entomol. 58:522524.

Taiz, L. and E. Zeiger. 1991. Cytokinins, p. 452472. In: L. Taiz and E. Zeiger (eds.). Plant physiology. Benjamin/Cummings, Redwood City, Calif.

Trumble, J.T., D.M. Kolodny-Hirsch, and I.P. Ting. 1993. Plant compensation for arthropod herbivory. Annu. Rev. Entomol. 38:93-119.

Wang, T.L., A.G. Thompson, and R. Horgan. 1977. A cytokinin glucoside from the leaves of Phaseolus vulgaris L. Planta 135:285-288.

Weaver, R.J. 1972. Plant growth substances in agriculture. Freeman, San Francisco. 